\title{
All that glisters is not an endophenotype: Rethinking endophenotypes in Anorexia Nervosa
}

\author{
Nadia Micali1 ${ }^{1,2} \&$ Camilla Lindvall Dahlgren ${ }^{1,3}$ \\ 1 Department of Psychiatry, Icahn School of Medicine at Mount Sinai, New York, \\ NY \\ 2 Institute of Child Health, University College London, London, UK \\ 3 Division of Mental Health and Addiction, Regional Department for Eating \\ Disorders, Oslo University Hospital, Oslo, Norway
}

Anorexia Nervosa (AN) is a serious mental health disorder with devastating consequences for sufferers and their families (1). AN has a peak of onset in early adolescence (2), an age of many developmental changes. AN with pre-pubertal onset (EO-AN) is rare, however the evidence is mixed on whether outcomes are worse or better compared to AN with onset after puberty. For many individuals AN becomes a chronic disorder, with impact on one's social, physical and psychological functioning. The last 10 years have seen many novel investigations focusing on the neurobiology of AN. Cognitive characteristics of patients with AN have received much attention, parallel to a surge of interest in psychiatry for measurable biological processes or phenotypes that might be more biologically informed than current diagnostic categories.

In this issue Van Noort and colleagues (3) aimed to investigate cognitive functioning in children with pre-pubertal AN, adolescents with $\mathrm{AN}$, and two groups of age matched controls. This design, i.e. including an age, gender and IQ matched control sample for each index group, allows taking into account agerelated changes in cognition-which are vast during this developmental stage. The authors suggest that the lack of differences in specific cognitive domains in children and adolescents with AN does not support the hypothesis of a cognitive endophenotype in AN.

Endophenotypes are 'internal phenotypes'; trait-like markers, that are thought to be more proximal to biological (or genetic) processes than a clinical phenotype (4). The definition of an endophenotype assumes that it should be: heritable, associated with the disorder, independent of clinical diagnosis (i.e. state independent), and found in family members who do not have the disorder at a higher level relative to the general population.

\section{Cognitive dysfunction as an Endophenotype in AN}

Over the past decade, studies in adults with AN have suggested that cognitive dysfunction in specific domains, e.g. central coherence and set-shifting, might be an AN endophenotype. The extant literature has yielded some support for this. Inefficiencies in central coherence and set-shifting meet some of the aforementioned endophenotype criteria. Adults with AN show differences in neuropsychological performance-based assessments compared to healthy controls $(5,6)$. Inefficiencies in both set-shifting and central coherence have been shown to manifest in unaffected family members of probands with AN (7). 
A twin study has also suggested a genetic contribution to both difficulties in global processing (i.e. central coherence) and in cognitive flexibility (e.g. setshifting). (8) However, less is known about whether these cognitive inefficiencies are independent of illness state (as required for the definition of an endophenotype), i.e. if these are present even in the absence of disorder.

\section{State-dependence - Age, weight and state of illness matter}

If cognitive weaknesses found to be associated with AN cross-sectionally are, in fact, state-independent, one would expect rigidity and inefficiencies in global information processing to be present before disease onset, across ages and in addition, to be unrelated to weight changes. Existing evidence points to the opposite. Firstly, several studies suggest that children and adolescents with AN do not exhibit the same weak cognitive profile as that of adults. Findings from van Noort et al., in this issue, provide similar evidence (3). In this study neither children with EO-AN nor adolescents with AN show central coherence, cognitive flexibility, planning, visuo-spatial skills, or cognitive inhibition inefficiencies compared to age, gender and IQ matched controls. Secondly, when it comes to changes associated with weight gain, studies have shown improvements in cognitive domains, such as attention, executive function and central coherence following weight gain $(9,10)$. Thirdly, mixed findings are present in relation to presence of cognitive weaknesses in central coherence and cognitive flexibility in subjects recovered from AN(11). Given this evidence, state-independence of cognitive rigidity and global processing difficulties in subjects with AN remains to be confirmed, challenging the hypothesis that these are AN endophenotypes.

\section{Alternative hypotheses and future steps}

Studying cognitive characteristics in individuals at risk for or prior to AN onset is difficult. There is a paucity of large enough longitudinal studies following individuals from before onset of disorder into the period of risk in the field of eating disorders. A study of children at risk for AN (familial) is currently under way and might shed some light onto cognitive and brain characteristics in the absence of active disorder. More research is needed into disentangling the effect of physical consequences of active AN, such as starvation, specific nutrient depletion, and hypoestrogenism, on brain structure and function from premorbid cognitive and brain dys-fucntion. Similarly, understanding possible longterm effects of the illness (scarring effects) on the brain and cognition might prove fruitful. AN is an heterogeneous disorder and the possibility that specific cognitive inefficiencies are present in a subgroup of individuals should not be ruled out. Although published genome-wide association studies (GWAS) of AN have not yielded positive findings, large genetic studies of AN are underway and these will provide robust markers of biological risk against which hypothesised endophenotypes can be tested.

\section{Conclusions}

Given the well known limitations of diagnostic categories in psychiatry, the quest for endophenotypes, especially across disorders, is in full swing. The NIMH Research Domain Criteria (RDoC) initiative has stimulated a focus on measurable processes that link biological and genetic liability to clinical disorders. Progress from GWAS in identifying genetic markers of disease will undoubtedly allow a 
better understanding of whether endophenotypes will stand the test of time in psychiatric research. We still know little about the neurobiology of AN; longitudinal, clinical and at-risk studies will likely inform our conceptualisation of this devastating disorder in the next ten years. This evidence will in the longterm allow refining existing treatment options and the likely development of new prevention and treatment strategies.

\section{References}

1. Herpertz-Dahlmann B, van Elburg A, Castro-Fornieles J, Schmidt U. ESCAP Expert Paper: New developments in the diagnosis and treatment of adolescent anorexia nervosa--a European perspective. Eur Child Adolesc Psychiatry. 2015;24(10):1153-67.

2. Micali N, Hagberg KW, Petersen I, Treasure JL. The incidence of eating disorders in the UK in 2000-2009: findings from the General Practice Research Database. BMJ Open. 2013;3(5).

3. van Noort BM, Pfeiffer E, Ehrlich S, Lehmkuhl U, Kappel V. Cognitive performance in children with acute early-onset anorexia nervosa. Eur Child Adolesc Psychiatry. 2016.

4. Gottesman, II, Gould TD. The endophenotype concept in psychiatry: etymology and strategic intentions. Am J Psychiatry. 2003;160(4):636-45.

5. Lang K, Lopez C, Stahl D, Tchanturia K, Treasure J. Central coherence in eating disorders: an updated systematic review and meta-analysis. World J Biol Psychiatry. 2014;15(8):586-98.

6. Roberts ME, Tchanturia K, Stahl D, Southgate L, Treasure J. A systematic review and meta-analysis of set-shifting ability in eating disorders. Psychol Med. 2007;37(8):1075-84.

7. Tenconi E, Santonastaso P, Degortes D, Bosello R, Titton F, Mapelli D, et al. Set-shifting abilities, central coherence, and handedness in anorexia nervosa patients, their unaffected siblings and healthy controls: exploring putative endophenotypes. World J Biol Psychiatry. 2010;11(6):813-23.

8. Kanakam N, Raoult C, Collier D, Treasure J. Set shifting and central coherence as neurocognitive endophenotypes in eating disorders: a preliminary investigation in twins. World J Biol Psychiatry. 2013;14(6):464-75.

9. Hatch A, Madden S, Kohn MR, Clarke S, Touyz S, Gordon E, et al. In first presentation adolescent anorexia nervosa, do cognitive markers of underweight status change with weight gain following a refeeding intervention? Int J Eat Disord. 2010;43(4):295-306.

10. Dahlgren CL, Lask B, Landro NI, Ro O. Neuropsychological functioning in adolescents with anorexia nervosa before and after cognitive remediation therapy: a feasibility trial. Int J Eat Disord. 2013;46(6):576-81.

11. Lindner SE, Fichter MM, Quadflieg N. Central coherence in full recovery of anorexia nervosa. Eur Eat Disord Rev. 2013;21(2):115-20. 